\title{
Nilvadipine Prevents the Impairment of Spatial Memory Induced by Cerebral Ischemia Combined with $\beta$-Amyloid in Rats
}

\author{
Katsunori Iwasaki, ${ }^{*, a, b}$ Nobuaki Egashira, ${ }^{a}$ Yuki Takagaki, ${ }^{a}$ Yoshitaka Yoshimitsu, ${ }^{a}$ \\ Izzettin Hatip-Al-KhatiB, ${ }^{c}$ Kenichi Mishima, ${ }^{a}$ and Michihiro FujIwARA ${ }^{a, b}$ \\ ${ }^{a}$ Department of Neuropharmacology, Faculty of Pharmaceutical Sciences, Fukuoka University; ${ }^{b}$ Advanced Material \\ Institute, Fukuoka University; 8-19-1 Nanakuma, Jonan-ku, Fukuoka 814-0180, Japan: and ' Department of \\ Pharmacology, Division of Internal Medicine, Faculty of Medicine, Pamukkale University; Denizli 20070, Turkey. \\ Received December 11, 2006; accepted January 22, 2007; published online January 23, 2007
}

In the present study, we examined the effects of nilvadipine and amlodipine, both dihydropyridine-derivative calcium antagonists, on the impairment of spatial memory induced by a combination of ischemia and $\beta$ amyloid $(\mathrm{A} \beta)$. Nilvadipine $(3.2 \mathrm{mg} / \mathrm{kg}$, i.p.) significantly prevented the impairment of spatial memory and neuronal apoptosis in this model. By contrast, amlodipine had no effect on this impairment of spatial memory. These findings suggest that nilvadipine may prevent impairment of spatial memory by inhibiting neuronal apoptosis; this drug might therefore be useful for the prevention of the progression to dementia in Alzheimer's disease (AD).

Key words Alzheimer's disease; $\beta$-amyloid; ischemia; nilvadipine; spatial memory; apoptosis

Dementia is one of the main symptoms in Alzheimer's disease (AD). In patients with sporadic $A D$, it has been reported that dementia appears at an early stage and is enhanced if patients show cerebrovascular disease (CVD) such as stroke. ${ }^{1)}$ A well known feature typical of $\mathrm{AD}$ is the accumulation of $\beta$-amyloid $(\mathrm{A} \beta)$ in senile plaques. ${ }^{2}$ ) The distribution of $\mathrm{A} \beta$ also correlates well with sites of neuronal damage that manifest as neurofibrillary tangles in brain regions such as the hippocampus, which is particularly vulnerable in AD and CVD. We previously reported that hypoxia, which is a key feature of ischemia, enhances $\mathrm{A} \beta$-induced apoptosis in rat cultured hippocampal neurons. ${ }^{3)}$ More recently, we reported that the combination of cerebral ischemia and $\mathrm{A} \beta$ administration, but not either treatment alone, impaired spatial memory and induced apoptosis of pyramidal neurons in the CA1 region of the hippocampus. ${ }^{4)}$ These findings suggest that ischemia is an important factor facilitating the symptoms of $\mathrm{AD}$; furthermore, this model might be useful for developing new drugs for the treatment of AD.

Nilvadipine is a dihydropyridine-derivative calcium antagonist, and a safe and effective agent prescribed for patients with both hypertension and chronic major cerebral artery occlusion. ${ }^{5}$ This drug's antioxidant effect is greater than that of nimodipine, nicardipine and amlodipine. ${ }^{6}$ ) Nilvadipine also reduces infarction in the middle cerebral artery occlusion model. ${ }^{7)}$ Moreover, we previously reported that nilvadipine, but not amlodipine, significantly reversed the impairment of spatial memory and hippocampal apoptosis induced by repeated cerebral ischemia. ${ }^{8)}$ Interestingly, nilvadipine has been reported to antagonize both $\mathrm{A} \beta$-induced vasoconstriction in isolated arteries and the reduced cerebral blood flow in an AD transgenic mouse model in which amyloid precursor protein (APP) is overexpressed (Tg 2576). ${ }^{9)}$ However, the effect of nilvadipine on memory impairment, in an animal model of $\mathrm{AD}$, remains unexplored. Accordingly, the present study was conducted to investigate the effect of nilvadipine, compared with amlodipine, on the impairment of spatial memory induced by a combination of cerebral ischemia and $\mathrm{A} \beta$.

\section{MATERIALS AND METHODS}

Animals Male Wistar rats, aged 7 weeks and weighing $200-250$ g, were obtained from Kyudo (Saga, Japan), and were housed in groups of four to five per cage in a room with the temperature controlled at $23 \pm 2{ }^{\circ} \mathrm{C}$, relative humidity of $60 \pm 10 \%$ and the lights on from 7:00 to $19: 00 \mathrm{~h}$. The animals were restricted in their food intake (10-12 g each day, CE-2; Clea Japan, Tokyo, Japan) and the body weight of each rat was maintained at $c a$. $80 \%$ of the freely feeding level. All animals had free access to drinking water in their home cages. All procedures were carried out at the Facilities for Experimental Animals, based on the regulations established by the Experimental Animal Care and Use Committee at Fukuoka University, Japan.

Behavioral Procedures for the Eight-Arm Radial Maze Behavioral testing was conducted as previously reported, ${ }^{8)}$ in an eight-arm radial maze (Neuroscience, Tokyo, Japan). The maze was elevated $50 \mathrm{~cm}$ above the floor. It consisted of a central platform $24 \mathrm{~cm}$ in diameter, with eight arms extending radially. Each arm was $50 \mathrm{~cm}$ long, $10 \mathrm{~cm}$ wide and $50 \mathrm{~cm}$ high with transparent plastic sides. Food cups for the reinforcers were placed near the end of each arm. The maze was located in a room containing many external visual cues. For the behavioral analysis, an image motion analyzer, AXIS-30 (Neuroscience), was used to quantify the task performance of rats in the eight-arm radial maze. This highspeed analyzer had an automatic tracking system which allowed the movement of each rat to be tracked in the maze with a charge-coupled devices camera equipped with a personal computer. A group of animals was trained so that they would become habituated to the apparatus and food pellets for $3 \mathrm{~d}$ before each test. A $10-$ min period of habituation was repeated three times a day, at intervals of $>1 \mathrm{~h}$. In each training session, the animal was placed within a circular plastic cage on the platform in the middle of the eight-arm radial maze. After $1 \mathrm{~min}$, the cage was lifted and the animal was allowed to move freely in the maze. The trial continued until the animal had either entered all eight arms or $10 \mathrm{~min}$ had elapsed. Animals that proceeded through the maze using 
non-spatial strategies, i.e., repeatedly choosing the arm adjacent to $\left(45^{\circ}\right)$ or the arm that was three arms away from $\left(135^{\circ}\right)$ the one currently visited, were excluded from the present experiment because they were considered to not have acquired spatial memory. The performance of the animal in each trial was assessed using two parameters: (1) the number of CC in the initial eight chosen arms, and (2) the number of EC which was defined as choosing arms which had already been visited. Only the rats which made no errors, or only one error, for three consecutive days were selected for the study.

Cerebral Ischemia Four-vessel occlusion was performed according to the method described in our previous study. ${ }^{8)}$ Briefly, rats were anesthetized with $50 \mathrm{mg} / \mathrm{kg}$ i.p. sodium pentobarbital and immobilized in stereotaxic apparatus. The bilateral vertebral arteries were electrocauterized with a bipolar coagulator (MICRO-3D; Mizuho Industrial Co., Tokyo, Japan). The bilateral common carotid arteries were then exposed and a hydraulic pressure vascular occluder (1.5 mm diameter, Technical Supply, Osaka, Japan) was applied to each exposed artery. The common carotid arteries were bilaterally compressed the next day with the hydraulic pressure occluders and cerebral circulation was interrupted for $10 \mathrm{~min}$. Body temperature was maintained at $37^{\circ} \mathrm{C}$ using a heating pad and lamp until recovery from anesthesia after surgical operations, or the righting reflex reappeared following occlusion of carotid arteries. Rats that did not exhibit loss of their righting reflex during arterial occlusion were excluded from the subsequent experiment. Rats that only underwent cauterization of the vertebral arteries, then had occluders fitted on the common carotid arteries, but without occlusion, were used as sham-operated controls.

Stereotaxic Procedure and $\mathbf{A} \boldsymbol{\beta}$ Injection Rats were anesthetized with sodium pentobarbital $(50 \mathrm{mg} / \mathrm{kg}$ i.p., Tokyo Kasei, Tokyo, Japan) and placed in a stereotaxic frame (Narishige Scientific Instruments, Tokyo, Japan) with the upper incisor bar $3.4 \mathrm{~mm}$ below the interaural line. Guide cannulae were implanted according to Paxinos and Watson. ${ }^{10)}$ For i.c.v. infusion, guide cannulae (o.d. $=0.71 \pm 0.02 \mathrm{~mm}$, i.d. $=0.41 \pm$ $0.02 \mathrm{~mm}$ and length $=13 \mathrm{~mm}$ ) were implanted bilaterally into the lateral cerebral ventricles at $\mathrm{AP}=-0.8 \mathrm{~mm}, \mathrm{~L}=1.3 \mathrm{~mm}$ and $\mathrm{H}=3.3 \mathrm{~mm}$. Implanted cannulae were anchored by two screws driven into the skull, fixed by dental acrylic, then secured with dummy cannulae kept in place by a cap. After surgery, each rat was injected with penicillin in the hindquarter muscle $(100000 \mathrm{U})$ and individually housed after operation. Body temperature was maintained using a heating pad and lamp during surgery and recovery from anesthesia. $\mathrm{A} \beta_{1-42} \mathrm{HCl}$ (ANASPEC, San Jose, CA, U.S.A.) was dissolved in sterile distilled water, and allowed to aggregate for $7 \mathrm{~d}$ at $37^{\circ} \mathrm{C}$. A $\beta_{1-42}$ injected daily for $7 \mathrm{~d}$ after cerebral ischemia. $\mathrm{A} \beta_{1-42}$ was administered bilaterally $(300 \mathrm{pmol} / 10 \mu \mathrm{l})$ using an injection cannula (o.d. $=0.35 \pm 0.01 \mathrm{~mm}$, i.d. $=0.17 \pm 0.02 \mathrm{~mm}$ and length $=14 \mathrm{~mm})$ connected by PEtubing (o.d. $=1.09 \mathrm{~mm}$, i.d. $=0.38 \mathrm{~mm}$; INTRAMEDIC; Becton Dickinson, MD, U.S.A.) to a perfusion pump (CMA/100, Microdialysis AB, Stockholm, Sweden) driven at rate of 1 $\mu \mathrm{l} / \mathrm{min}$. The injection cannulae protruded $1 \mathrm{~mm}$ beyond the guide cannulae. The behavioral test was performed $60 \mathrm{~min}$ after last injection of $\mathrm{A} \beta_{1-42}$

Drug Testing The behavioral test was performed $60 \mathrm{~min}$ after last injection of $\mathrm{A} \beta_{1-42}$. Nilvadipine and amlodipine
(Astellas Pharma Inc., Tokyo, Japan) were dissolved in $25 \%$ polyethylene glycol and injected intraperitoneally $30 \mathrm{~min}$ prior to $\mathrm{A} \beta$ treatment.

Apoptosis Detection We investigated the involvement of the apoptosis in the effect of nilvadipine on impairment of spatial memory. By contrast, we did not examine on the incidence of apoptosis in amlodipine-treated rats because of amlodipine had no effect on memory impairment.

After completion of the behavioral experiment (one week after last injection of $\mathrm{A} \beta_{1-42}$ ), animals were euthanized by deep sodium pentobarbital $(50 \mathrm{mg} / \mathrm{kg}$, i.p.) anesthesia and perfused transcardially with heparinized saline followed by 4\% paraformaldehyde. Brains were removed, fixed overnight in paraformaldehyde and stored in phosphate buffer, before being cut into $10 \mu \mathrm{m}$ sections. Each section was stained for apoptotic cells using the terminal deoxynucleotidyl transferase (TdT)-mediated fluorescein-deoxyuridinetriphosphate (dUTP) nick-end labeling (TUNEL) technique (TACSII, Trevigen, MD, U.S.A.). Propidium iodide ( $1 \mu \mathrm{g} / \mathrm{ml}$, red, Sigma) was used for counter staining. Fluorescein-dUTP-labeled fragmented DNA can be visualized directly by fluorescence microscopy (Leica, DMRA Germany). TUNEL-positive cells (green; FITC) in sections that included the CA1 field of the dorsal hippocampus were counted.

Statistical Analyses Radial maze performance and the number of apoptotic cells were evaluated using the one-way analysis of variance (ANOVA) followed by the Fisher's PLSD post-hoc test. The criterion for statistical significance was considered to be $p<0.05$. Values are expressed as the mean \pm S.E.M

\section{RESULTS}

Effects of Nilvadipine and Amlodipine on the Combination of Ischemia and $A \boldsymbol{\beta}$-Induced Impairment of Spatial Memory Figure 1 shows that the combination of ischemia and $\mathrm{A} \beta$ produced a marked impairment of spatial memory $(\mathrm{CC}: F(3,34)=8.858, p<0.001$; EC: $F(3,34)=$ $4.488, p<0.01$, one-way ANOVA), significantly decreasing the number of $\mathrm{CC}(6.1 \pm 0.2)$ and increasing the number of EC $(5.4 \pm 1.0)$ compared to the sham group (CC: $7.6 \pm 0.2$; EC: $0.7 \pm 0.3)(p<0.01$ by the Fisher's PLSD post-hoc test). Nilvadipine at the dose of $3.2 \mathrm{mg} / \mathrm{kg}$ significantly reversed

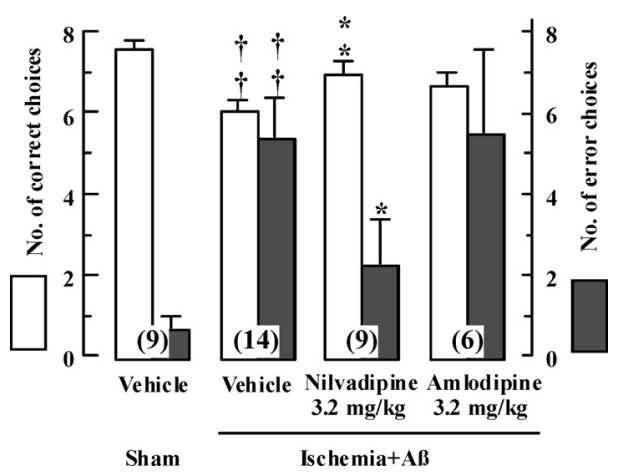

Fig. 1. Effects of Nilvadipine and Amlodipine on the Impairment of Spatial Memory Induced by Cerebral Ischemia Combined with $\mathrm{A} \beta$

Nilvadipine and amlodipine were administered $30 \mathrm{~min}$ prior to $\mathrm{A} \beta$ treatment values are expressed as the mean \pm S.E.M. ${ }^{\dagger} p<0.01$ compared to the sham group, $* p<0.05$, $* * p<0.01$ compared to the vehicle group. The number of rats is shown at the bottom of each column. Abbreviations; $\mathrm{A} \beta, \beta$-amyloid. 


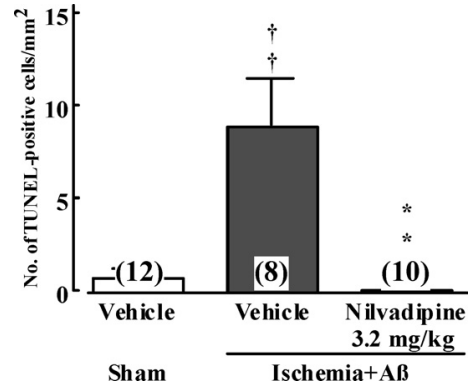

Fig. 2. Effect of Nilvadipine on the Number of Apoptotic Cells in the Ca1 Area of the Hippocampus, Following Cerebral Ischemia Combined with A $\beta$

Values are expressed as the mean \pm S.E.M. ${ }^{\dagger} p<0.01$ compared to the sham group, $* * p<0.01$ compared to the vehicle group. The number of rats is shown at the bottom of each column. Abbreviations; $\mathrm{A} \beta, \beta$-amyloid.

the impairment of spatial memory induced by ischemia combined with $\mathrm{A} \beta$ (CC: $7.0 \pm 0.3, p<0.01 ; \mathrm{EC}: 2.3 \pm 1.1, p<0.05$ by the Fisher's PLSD post-hoc test). By contrast, amlodipine had no effect on this impairment of spatial memory (CC: $6.7 \pm 0.3, p>0.05$; EC: $5.5 \pm 2.1, p>0.1$ by the Fisher's PLSD post-hoc test).

Effect of Nilvadipine on the Combination of Ischemia and $\mathbf{A} \boldsymbol{\beta}$-Induced Hippocampal Apoptosis The combination of ischemia and $\mathrm{A} \beta$ significantly increased the number of apoptotic cells $(8.5 \pm 2.5)$ compared to the sham group $(0.7 \pm 0.4)(p<0.01$ by the Fisher's PLSD post-hoc test, Fig. $2)$. Nilvadipine significantly reduced the number of apoptotic cells $(0.0 \pm 0.0, p<0.01$ by the Fisher's PLSD post-hoc test $)$.

\section{DISCUSSION}

In the present study, we found that nilvadipine (but not amlodipine) prevented impairment of spatial memory induced by cerebral ischemia combined with $\mathrm{A} \beta$. Moreover, nilvadipine markedly reduced the neuronal apoptosis in this model. Therefore, the protection of spatial memory seen with nilvadipine might be due to its inhibition of neuronal apoptosis. In fact, we previously found that nilvadipine $(3.2 \mathrm{mg} / \mathrm{kg}$, i.p.) did not improve the impairment of spatial memory induced by a single cerebral ischemic event, which does not induce apoptosis. ${ }^{8)}$ On the other hand, nilvadipine (but not amlodipine) at the same dose improved the repeated cerebral ischemia-induced impairment of spatial memory, concomitantly decreased hippocampal apoptosis, and suppressed both bax and CPP 32 (caspase-3) expression. ${ }^{8} \mathrm{Ca}^{2+}$ is an important regulator of both cell survival and apoptosis in response to a plethora of pathological insults. ${ }^{11)}$ Nilvadipine, by strongly blocking $\mathrm{Ca}^{2+}$ influx through L- and T-type voltagegated $\mathrm{Ca}^{2+}$ channels in the hippocampal CAl field, ${ }^{12)}$ controls intracellular $\mathrm{Ca}^{2+}$ ion cascades and accordingly could prevent apoptosis and provide protection to neurons. However, the effect on $\mathrm{Ca}^{2+}$ channels is not the sole mechanism involved in the activity of nilvadipine, because nilvadipine did not improve single ischemia-induced memory impairment which involves extensive $\mathrm{Ca}^{2+}$ overload. ${ }^{8)}$ Moreover, amlodipine, a L-type $\mathrm{Ca}^{2+}$ channel blocker, had no effect on the impairment of spatial memory induced by cerebral ischemia combined with $\mathrm{A} \beta$. The caspase enzymes, which were suppressed by nilvadipine in the repeated cerebral is- chemia model, modulate intracellular $\mathrm{Ca}^{2+}$ dynamics by directly interacting with channels and transporters or forming pores, and thus, regulate cellular $\mathrm{Ca}^{2+}$ levels. ${ }^{11)}$ The apoptosis cascade initiated by ischemia-induced $\mathrm{Ca}^{2+}$ influx might be modulated by a death signal produced by membrane-binding of aggregated $\mathrm{A} \beta$, leading to overt neuronal injury. Recent reports have demonstrated that caspases, key enzymes that promote apoptosis, are involved in the proteolysis of APP and resultant accumulation of $\mathrm{A} \beta,{ }^{13)}$ and suggest that $\mathrm{A} \beta$ itself induces apoptosis via activation of caspase-8. ${ }^{14}$ These findings suggest the possibility that $\mathrm{A} \beta$ is directly involved in induction of apoptosis. Accordingly, it could be suggested that nilvadipine prevents the neuronal apoptosis not only by antagonizing $\mathrm{Ca}^{2+}$ channels directly, but also via mechanisms mediated by apoptosis-related caspase enzymes. On the other hand, recent literature suggests that there is a caspase-independent apoptosis-like cell death. ${ }^{15)}$ Therefore, caspase-independent pathway may also be involved in the neuronal apoptosis induced by cerebral ischemia combined with $\mathrm{A} \beta$.

In the present study, nilvadipine but not amlodipine prevented the impairment of spatial memory induced by a combination of ischemia and $\mathrm{A} \beta$. Amlodipine has been reported to exert a very potent neuroprotective effect and to inhibit free radical-induced damage to the membranous lipid constituents of rat cerebellar granule cells in vitro. ${ }^{16)}$ However, it had no effect on this model. This result could be due to the poor penetration of the blood brain barrier, ${ }^{17)}$ low antioxidant and anti-caspase activities.

In conclusion, nilvadipine prevents the impairment of spatial memory and neuronal apoptosis induced by cerebral ischemia combined with $\mathrm{A} \beta$. These findings suggest that nilvadipine might be a useful drug for the prevention of progression to dementia in AD.

Acknowledgments We thank Astellas Pharma Inc. for the generous supply of nilvadipine and amlodipine. This study was supported by the Advanced Material Institute, University of Fukuoka and a Grant-in-Aid for Scientific Research \#13672407 from the Ministry of Education, Culture, Sports, Science and Technology of Japan.

\section{REFERENCES}

1) Snowdon D. A., Greiner L. H., Mortimer J. A., Riley K. P., Greiner P. A., Markesbery W. R., JAMA, 277, 813-817 (1997).

2) Selkoe D. J., Annu. Rev. Neurosci., 12, 463-490 (1989).

3) Egashira N., Iwasaki K., Ishibashi M., Hatip-Al-Khatib I., Wolozin B., Mishima K., Irie K., Fujiwara M., Jpn. J. Pharmacol., 90, 321-327 (2002).

4) Iwasaki K., Egashira N., Hatip-Al-Khatib I., Akiyoshi Y., Arai T., Takagaki Y., Watanabe T., Mishima K., Fujiwara M., Brain Res., 1097, $216-223$ (2006).

5) Ogasawara K., Noda A., Yasuda S., Kobayashi M., Yukawa H., Ogawa A., Nucl. Med. Commun., 24, 71-76 (2003).

6) Sugawara H., Tobise K., Kikuchi K., Hypertens. Res., 19, 223-228 (1996).

7) Li Y., Kawamura S., Yasui N., Shirasawa M., Fukasawa H., Exp. Brain Res., 99, 1-6 (1994).

8) Iwasaki K., Mishima K., Egashira N., Hatip-Al-Khatib I., Ishibashi D., Irie K., Kobayashi H., Egawa T., Fujiwara M., J. Pharmacol. Sci., 93, 188-196 (2003).

9) Paris D., Quadros A., Humphrey J., Patel N., Crescentini R., Crawford F., Mullan M., Brain Res., 999, 53-61 (2004). 
10) Paxinos G., Watson C., "The Rat Brain Stereotaxic Coordinates," Academic Press, New York, 1982.

11) Hajnoczky G., Davis E., Madesh M., Biochem. Biophys. Res. Commun., 304, 445-454 (2003).

12) Ishibashi H., Murai Y., Akaike N., Brain Res., 813, 121-127 (1998).

13) Gervais F. G., Xu D., Robertson G. S., Vaillancourt J. P., Zhu Y., Huang J., LeBlanc A., Smith D., Rigby M., Shearman M. S., Clarke E. E., Zheng H., Van Der Ploeg L. H., Ruffolo S. C., Thornberry N. A., Xanthoudakis S., Zamboni R. J., Roy S., Nicholson D. W., Cell, 97,
395-406 (1999).

14) Ivins K. J., Thornton P. L., Rohn T. T., Cotman C. W., Neurobiol. Dis., 6, 440-449 (1999).

15) Kroemer G., Martin S. J., Nat. Med., 11, $725-730$ (2005).

16) Mason R. P., Leeds P. R., Jacob R. F., Hough C. J., Zhang K. G., Mason P. E., Chuang D. M., J. Neurochem., 72, 1448-1456 (1999).

17) Uchida S., Yamada S., Nagai K., Deguchi Y., Life Sci., 61, 20832090 (1997). 